\title{
A Nationwide Survey in Japan of Palliative Radiotherapy for Bleeding in Gastrointestinal and Genitourinary Tumor Patients
}

\author{
Takashi Kosugi ${ }^{a}$, , Naoto Shikama ${ }^{b}$, Tetsuo Saito ${ }^{c}$, Naoki Nakamura $^{\mathrm{d}}$, Ayako Nakura ${ }^{\mathrm{a}}$, \\ Hideyuki Harada ${ }^{\mathrm{e}}$, Hitoshi Wada ${ }^{\mathrm{f}}$, Miwako Nozaki ${ }^{\mathrm{g}}$, Nobue Uchida ${ }^{\mathrm{h}}$, Katsumasa Nakamura ${ }^{\mathrm{i}}$
}

\begin{abstract}
Background: In end-stage cancer, bleeding may markedly influence the patient's quality of life, and radiotherapy plays an important role in the control of the bleeding. In particular, there is no clear evidence of the benefit of palliative radiotherapy for cancers including gastric, rectal, and bladder cancers. A clarification of the current status of palliative radiotherapy for bleeding is needed.
\end{abstract}

Methods: A survey was conducted by the palliative radiotherapy working group of the Japanese Radiation Oncology Study Group (JROSG), focusing on annual cases of radiotherapy for tumors of the upper and lower gastrointestinal tracts and genitourinary organs (excluding the uterus) and applicable fractionated doses for three hypothetical patients.

Results: A total of 54 radiation oncologists at 43 facilities answered. Most of the facilities reported that they conducted hemostatic irradiation for less than one patient per year, though the median annual number of patients treated with radiotherapy in these facilities was

Manuscript accepted for publication June 23, 2016

aDepartment of Radiation Oncology, Fujieda Municipal General Hospital, Fujieda, Shizuoka, Japan

${ }^{b}$ Department of Radiation Oncology, Saitama Medical University International Medical Center, Hidaka, Saitama, Japan

'Department of Radiation Oncology, Kumamoto University Hospital, Kumamoto, Kumamoto, Japan

dDepartment of Radiation Oncology, National Cancer Center Hospital East, Kashiwa, Chiba, Japan

eDivision of Radiation Oncology, Shizuoka Cancer Center, Nagaizumi, Sunto, Shizuoka, Japan

fDepartment of Radiation Oncology, Southern TOHOKU General Hospital, Koriyama, Fukushima, Japan

gDepartment of Radiation Oncology, Dokkyo Medical University Koshigaya Hospital, Koshigaya, Saitama, Japan

hDepartment of Radiation Oncology, Tottori University Hospital, Yonago, Tottori, Japan

'Department of Radiation Oncology, Hamamatsu University School of Medicine, Hamamatsu, Shizuoka, Japan

${ }^{j}$ Corresponding Author: Takashi Kosugi, Department of Radiation Oncology, Fujieda Municipal General Hospital, 4-1-11, Surugadai, Fujieda, Shizuoka 426-8677, Japan. Email: tkskosugi@gmail.com

doi: http://dx.doi.org/10.14740/wjon977w
594. The most frequently observed doses per fraction for two of the hypothetical cases were 3.0 and 2.0 Gy. On the other hand, in each case, the numbers of fractions reported varied markedly among the respondents, ranging from $20 \mathrm{~Gy}$ in five fractions to over 30 fractions.

Conclusions: Especially in gastric cancer, hemostatic irradiation is rarely performed in most facilities in Japan. An optimal dose fractionation has not been established. Thirty Gy in 10 fractions is one of the most frequently used regimens, but re-bleeding was observed in approximately one-third of the patients.

Keywords: Palliative radiotherapy; Bleeding; Hemostasis; Survey

\section{Introduction}

In end-stage cancer, bleeding that requires a blood transfusion may markedly influence the patient's quality of life. Bleeding control is thus an important issue, and radiotherapy plays an important role in the control of the bleeding [1]. The usefulness of palliative thoracic irradiation for symptoms such as hemoptysis, cough, chest pain, and dyspnea has been confirmed in a systematic review of 13 randomized controlled trials by Fairchild et al [2]. They concluded that, by using a tumor alpha/ beta ratio of 10, an at least biologically equivalent dose (BED) of $35 \mathrm{~Gy}_{10}$ may be warranted. However, there was no similar clear evidence for other cancers including gastric, rectal, and bladder cancers, and the effectiveness of hemostatic irradiation for these tumors has not been well evaluated $[3,4]$.

Gastric cancer is one of the most common malignancies in Japan. However, regarding hemostatic irradiation, only a few retrospective reports with small cohorts of patients have been published in Japan [5-7], whereas relatively large numbers of patients with gastric cancer who were treated with palliative hemostatic irradiation have been evaluated in other Asian countries $[8,9]$. Although the response rates were promising [10], optimal regimens for hemostatic radiotherapy of patients with gastrointestinal (GI) tract tumors or genitourinary (GU) malignancies have not been established. To clarify the current status and the patterns of palliative radiotherapy for tumor-related bleeding in Japan, a nationwide survey was conducted by the Japanese Radiation Oncology Study Group 
Table 1. The Survey's Three Hypothetical Patients With GI or GU Cancer

Case 1 A 65-year-old male with ECOG performance status 1 who was diagnosed with gastric cancer involving multiple hepatic metastases 6 months ago. His response to chemotherapy has been poor, and the disease is progressing. The patient requires blood transfusion approximately once weekly due to bleeding from the primary lesion. There are no alternative hemostatic approaches.

Case 2 A 75-year-old female with ECOG performance status 2 who was diagnosed with rectal cancer involving multiple hepatic and pulmonary metastases 1 year earlier. She has undergone multiple chemotherapy sessions. The continuation of chemotherapy is difficult due to myelosuppression. The presence of hematochezia involving bleeding from the primary lesion in the rectum has been macroscopically confirmed. Although blood transfusion is not required at present, the hemoglobin level is decreasing. There are no alternative hemostatic approaches.

Case 3 An 80-year-old male with ECOG performance status 1 who was diagnosed with bladder cancer involving bone 1 month earlier. Chemotherapy has not been applied in consideration of the patient's age and complication by ischemic heart disease and others. The presence of macroscopic hematuria suggests bleeding from the bladder tumor. Mild anemia is also observed, not requiring blood transfusion at present.

ECOG: Eastern Cooperative Oncology Group.

(JROSG).

\section{Materials and Methods}

The JROSG Palliative Radiotherapy Working Group created a survey questionnaire. The JROSG is comprised of radiation oncologists in Japan, and most of the country's large faculties including university hospitals and cancer centers have participated in this group. The survey was sent by e-mail to all of the JROSG members in 115 faculties on October 6, 2014, and the deadline for replying was the end of that month.

The approximate annual numbers of patients treated with radiotherapy in each facility were derived from the institutional database reported to the JROSG office between 2006 and 2013.

The survey asked for the total numbers of patients treated with hemostatic irradiation for the tumors of the GI tract (esophagus, stomach, and duodenum) and GU organs (kidney, ureter, bladder, and prostate) in the calendar year 2013. Uterine cancers were excluded from this survey, because they may be treated by brachytherapy. The respondents also indicated the total dose and fractions they would recommend for three hypothetical bleeding cases with advanced tumors (Table 1). Case 1 was an advanced gastric cancer patient with multiple liver metastases and bleeding from the primary lesion. The tumors were chemotherapy-resistant, and a blood transfusion was required. Case 2 was an advanced rectal cancer patient with multiple lung and liver metastases in disease progression after systemic chemotherapy. The patient suffered from a small amount of bleeding, but blood transfusion was not indicated. Case 3 had bladder cancer with a single bone metastasis. Blood transfusion was not indicated, and radiotherapy was recommended as the initial therapy because of the patient's advanced age. The authors could not receive any patient's personally identifiable information from respondents. Of course, the radiation oncologists who agreed with this survey answered.

\section{Results}

A total of 54 radiation oncologists at 43 facilities (37.4\% of the JROSG facilities) responded. Of those respondents, 36 $(66.7 \%)$ worked at university hospitals or cancer centers, 15 $(27.8 \%)$ at public hospitals, and three (5.6\%) at private hospitals. The median annual number of patients treated with radiotherapy among the 43 facilities was 594 (range 145 - 1,800).

The numbers of the patients treated with hemostatic irradiation per year at each institution are shown in Figure 1. Hemostatic irradiation for tumors in the upper or lower GI tracts was rarely performed in most of the institutions, and more patients with GU tumors tended to be treated with hemostatic irradiation compared to the patients with GI tumors.

The dose fractionations indicated for the three hypothetical cases markedly varied among the survey respondents (Table 2 and Fig. 2). A total of 23 dose fractionations were extracted, ranging from $25 \mathrm{~Gy}$ in five fractions to $59.4 \mathrm{~Gy}$ in 33 fractions.

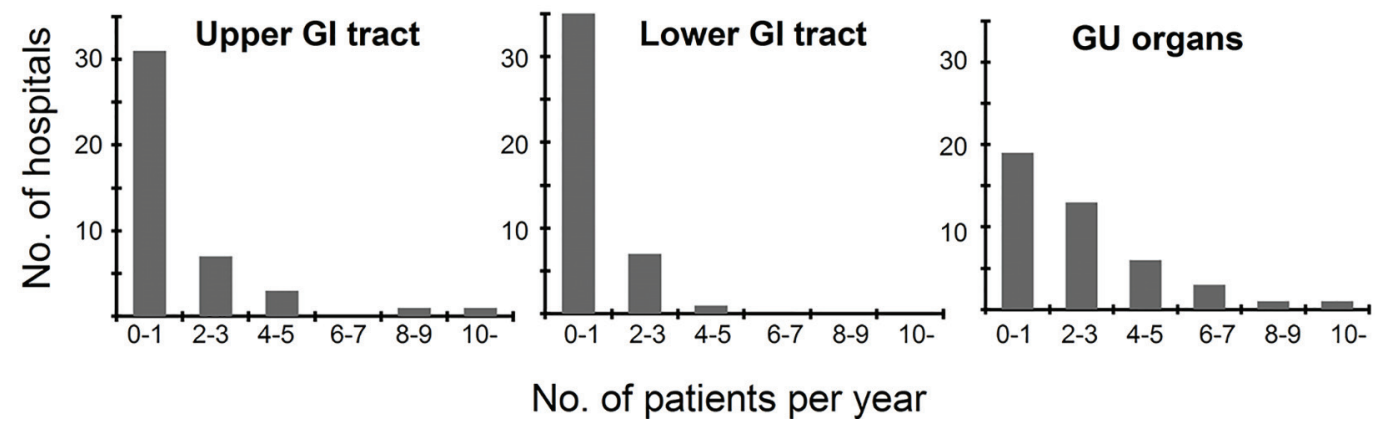

Figure 1. Annual numbers of patients with tumor-related bleeding treated with hemostatic irradiation. 
Table 2. Dose Schedules Submitted for Cases of Bleeding (n $=54)^{*}$

\begin{tabular}{|c|c|c|}
\hline $\begin{array}{l}\text { Dose/fraction } \\
\text { (Gy) }\end{array}$ & n (\%) & $\begin{array}{l}\text { Median no. of } \\
\text { fractions (range) }\end{array}$ \\
\hline \multicolumn{3}{|l|}{ Case 1} \\
\hline$>3.0$ & $3(4.8)$ & 5 \\
\hline 3.0 & $33(52.4)$ & $10(10-15)$ \\
\hline 2.5 & $9(14.3)$ & $16(14-18)$ \\
\hline 2.4 & $1(1.6)$ & 15 \\
\hline 2.0 & $16(26.7)$ & $20(10-30)$ \\
\hline 1.8 & $1(1.6)$ & 25 \\
\hline No indication & 0 & \\
\hline Total & 63 & $10(5-30)$ \\
\hline \multicolumn{3}{|l|}{ Case 2} \\
\hline$>3.0$ & $1(1.7)$ & 5 \\
\hline 3.0 & $33(55.0)$ & $10(10-18)$ \\
\hline 2.5 & $9(15.0)$ & $16(16-20)$ \\
\hline 2.4 & $0(0)$ & \\
\hline 2.0 & $14(23.3)$ & $25(15-30)$ \\
\hline 1.8 & $3(5.0)$ & $28(25-33)$ \\
\hline No indication & $3(5.0)$ & \\
\hline Total & 60 & $14(5-33)$ \\
\hline \multicolumn{3}{|l|}{ Case 3} \\
\hline$>3.0$ & $2(3.3)$ & $7.5(5-10)$ \\
\hline 3.0 & $16(26.7)$ & $10(10-15)$ \\
\hline 2.5 & $9(15.0)$ & $20(16-20)$ \\
\hline 2.4 & 0 & \\
\hline 2.0 & $29(48.3)$ & $25(20-30)$ \\
\hline 1.8 & $2(3.3)$ & $29(28-30)$ \\
\hline No indication & $2(3.3)$ & \\
\hline Total & 60 & $20(5-30)$ \\
\hline
\end{tabular}

*Since multiple answers were allowed, the data do not necessarily add up to 54 .

The most frequently recommended treatment was 30 Gy in 10 fractions (BED of $39 \mathrm{~Gy}_{10}$ ) for cases 1 and 2. Interestingly, more respondents selected slightly higher BED regimens for case 2 compared to the number of respondents selecting such regimens for case 1 . On the other hand, for case 3 (who had an oligometastatic bladder cancer), the most recommended fraction dose was 2.0 Gy per fraction, and most of the respondents prescribed higher BED regimens than 39 Gy10. Three and two respondents answered that radiotherapy was not applicable in cases 2 and 3 , respectively, for whom blood transfusion was not indicated.

\section{Discussion}

The median annual number of patients treated with radiother- apy among the facilities in this survey was 594. The average annual number of new cancer patients treated in a single Japanese radiotherapy facility was reported to be 270 patients in 2010 [11], and most of the respondents in this survey belonged to large institutions in Japan. Nevertheless, our results revealed that the numbers of patients with GI tract or GU tumors treated with hemostatic irradiation were very small in Japan, and the fractionation regimens varied markedly among the respondents.

As for gastric cancer, hemostatic irradiation for tumorassociated bleeding is rarely performed in Japan (Fig. 1), although gastric cancer is one of the most common malignancies here. The first choice of treatment for tumor bleeding from inoperable gastric cancer may be endoscopic management [12]. However, re-bleeding after successful hemostasis with endoscopic therapy frequently occurs; thus endoscopic hemostasis is often unsuccessful as a way to stop bleeding, and radiotherapy should be considered as the next step [5-7]. Asakura et al reported that 30 patients with advanced gastric cancer requiring blood transfusion were treated with a total of $30 \mathrm{~Gy}$ at 3 Gy per fraction, and the response rate was 74\%. Kondoh also demonstrated that $73 \%$ of 17 patients with advanced gastric cancer achieved hemostasis after a median dose of 30 Gy in 10 fractions. Tey et al retrospectively reviewed a large series of symptomatic gastric cancer patients treated with radiotherapy in Singapore, and they reported that a favorable hemostatic effect was obtained in $80.6 \%$ of 115 cases [8]. Interestingly, various fractionation regimens were described in their report, from 8 Gy single fractions to 40 Gy in 16 fractions [8].

Cameron et al reviewed 27 studies on palliative pelvic radiotherapy for symptomatic rectal cancer, and positive responses were reported for $81 \%$ of the cases of bleeding and discharge, although there were large variations in the applied radiotherapy regimens [4]. They demonstrated that the most commonly used total doses were $30-60 \mathrm{~Gy}$. This tendency is consistent with our results shown in Figure 2. Duchesne et al also reported that $68 \%$ of their patients showed the improvement of local symptoms including hematuria from muscle-invasive bladder cancer, with the use of two radiotherapy schedules (35 Gy in 10 fractions and 21 Gy in three fractions) [13]. These reports indicated that radiotherapy should be considered as one choice for tumor-associated bleeding.

Optimal fraction regimens for hemostatic radiotherapy have not yet been determined. Figure 2 shows that the numbers of fractions used varied greatly among our survey respondents, and the most frequently observed dose per fraction was $30 \mathrm{~Gy}$ in 10 fractions with palliative intent (cases 1 and 2). Previous studies also support these tendencies [5-7]. However, it should be noted that re-bleeding after radiotherapy occurred at a certain rate. Kondoh et al reported that re-bleeding was observed in $36 \%$ of 11 patients who achieved hemostasis after radiotherapy with 30 Gy in 10 fractions [7]. Asakura et al demonstrated that re-bleeding occurred in $50 \%$ of 22 patients with advanced gastric cancer responding to hemostatic radiotherapy using 30 Gy at 3 Gy per fraction [5]. They also reported that the rate of patients free from re-bleeding was lower in their chemoradiotherapy group than the radiotherapy alone group. Hashimoto et al observed that a BED of $50 \mathrm{~Gy}_{10}$ or more was significantly correlated with successful hemostasis for unresectable gastric 

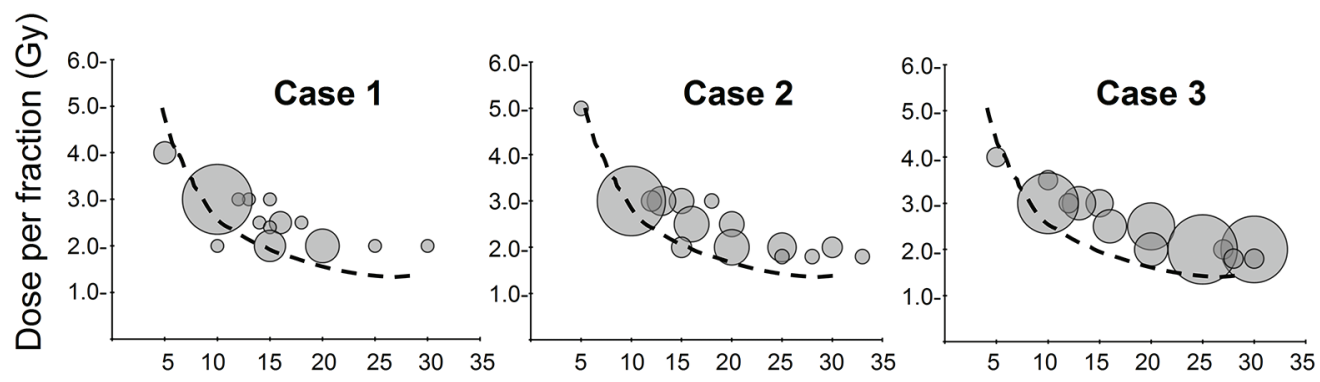

No. of fractions

Figure 2. Relationship between dose per fraction and number of fractions. The bubble size represents the number of answers. The dashed line indicates the biologically equivalent dose (BED) of $39 \mathrm{~Gy}_{10}$ by using a tumor alpha/beta ratio of 10 .

cancer [6]. Although a fractionation regimen of 30 Gy in 10 fractions is commonly used for palliative radiotherapy [14], this fractionation regimen may be less effective to maintain hemostasis for a certain period.

Dose fractionation is influenced by multiple factors, of which one of the most powerful is the prognosis. As shown in Figure 2, most of the survey respondents selected higher $\mathrm{BED}$ regimens than $39 \mathrm{~Gy}_{10}$ for case 3 , who had bladder cancer with single bone metastasis. Fairchild et al pointed out that the prognosis is the most important influencing factor [15]. In the present study's survey, the greatest number of radiation oncologists selected the response "a lot" for determining the dose fractionation schedule against bone metastasis. The maintenance of a favorable balance between the prognosis and duration of treatment is a challenge. A limited prognosis tends to be subjectively estimated by the physician(s) in charge, and this may be one of causes why various fractionation regimens are used. A large number of reports have discussed the usefulness of scales including the palliative prognostic scale to estimate the prognosis of advanced cancer patients [16]. If more accurate prognostication can be achieved, it may be easier to standardize the fractionation regimens for hemostatic irradiation.

The necessity of blood transfusion is also an influencing factor. A few respondents considered that radiotherapy was not applicable in cases 2 and 3, for whom blood transfusion was not indicated, whereas radiotherapy was regarded as applicable by all respondents. Radiation oncologists should bear in mind that patients with tumor-related bleeding that requires a blood transfusion are good candidates for hemostatic irradiation.

To the best of our knowledge, this is the first survey on hemostatic radiotherapy. Although hemostatic irradiation was rarely used among the survey respondents, and in particular, the effectiveness of hemostatic irradiation for tumors in the GI tracts and the optimal fractionation regimens remain unclear, the efficacy of hemostatic irradiation has been reported. Although important information has been obtained through this survey as a preliminary step, prospective studies are necessary for the determination of optimal radiation doses.

\section{Acknowledgments}

The authors wish to acknowledge all of the participants who responded to our survey.

\section{Conflicts of Interest}

The authors have no conflicts of interest to disclose with respect to this article.

\section{References}

1. Pereira J, Phan T. Management of bleeding in patients with advanced cancer. Oncologist. 2004;9(5):561-570.

2. Fairchild A, Harris K, Barnes E, Wong R, Lutz S, Bezjak A, Cheung $\mathrm{P}$, et al. Palliative thoracic radiotherapy for lung cancer: a systematic review. J Clin Oncol. 2008;26(24):4001-4011.

3. Cameron MG, Kersten C, Vistad I, Fossa S, Guren MG. Palliative pelvic radiotherapy of symptomatic incurable rectal cancer - a systematic review. Acta Oncol. 2014;53(2):164-173.

4. Cameron MG, Kersten C, Guren MG, Fossa SD, Vistad I. Palliative pelvic radiotherapy of symptomatic incurable prostate cancer - a systematic review. Radiother Oncol. 2014;110(1):55-60.

5. Asakura H, Hashimoto T, Harada H, Mizumoto M, Furutani K, Hasuike N, Matsuoka M, et al. Palliative radiotherapy for bleeding from advanced gastric cancer: is a schedule of 30 Gy in 10 fractions adequate? J Cancer Res Clin Oncol. 2011;137(1):125-130.

6. Hashimoto K, Mayahara H, Takashima A, Nakajima TE, Kato K, Hamaguchi T, Ito Y, et al. Palliative radiation therapy for hemorrhage of unresectable gastric cancer: a single institute experience. J Cancer Res Clin Oncol. 2009;135(8):1117-1123.

7. Kondoh C, Shitara K, Nomura M, Takahari D, Ura T, Tachibana H, Tomita N, et al. Efficacy of palliative radiotherapy for gastric bleeding in patients with unresectable advanced gastric cancer: a retrospective cohort study. BMC Palliat Care. 2015;14:37.

8. Tey J. Choo B, Leong C. Clinical Outcome of Palliative Radiation Therapy in Locally Advanced, Symptomatic Gastric Cancer in the Modern Era. Medicine (Baltimore). 
2014;93:1-6.

9. Chaw CL, Niblock PG, Chaw CS, Adamson DJ. The role of palliative radiotherapy for haemostasis in unresectable gastric cancer: a single-institution experience. Ecancermedicalscience. 2014;8:384.

10. Furuya Y, Akakura K, Akimoto S, Ichikawa T, Ito H. Radiotherapy for local progression in patients with hormonerefractory prostate cancer. Int J Urol. 1999;6(4):187-191.

11. http://www.jastro.or.jp/cmsdesigner/dlfile. php? entryname $=$ aboutus_child\&entryid $=00038 \&$ file $\mathrm{id}=00000002 \& / A R R 111 . p \overline{d f}$ (Accessed Dec 2015)

12. Kim YI, Choi IJ. Endoscopic management of tumor bleeding from inoperable gastric cancer. Clin Endosc. 2015;48(2):121-127.

13. Duchesne GM, Bolger JJ, Griffiths GO, Trevor Roberts J, Graham JD, Hoskin PJ, Fossa SD, et al. A randomized trial of hypofractionated schedules of palliative radiotherapy in the management of bladder carcinoma: results of medical research council trial BA09. Int J Radiat Oncol Biol Phys. 2000;47(2):379-388.

14. Nakamura N, Shikama N, Wada H, Harada H, Nozaki M, Nagakura H, Tago M, et al. Patterns of practice in palliative radiotherapy for painful bone metastases: a survey in Japan. Int J Radiat Oncol Biol Phys. 2012;83(1):e117-120.

15. Fairchild A, Barnes E, Ghosh S, Ben-Josef E, Roos D, Hartsell W, Holt T, et al. International patterns of practice in palliative radiotherapy for painful bone metastases: evidence-based practice? Int J Radiat Oncol Biol Phys. 2009;75(5):1501-1510.

16. Lau F, Downing GM, Lesperance M, Shaw J, Kuziemsky C. Use of Palliative Performance Scale in end-of-life prognostication. J Palliat Med. 2006;9(5):1066-1075. 